\title{
ANÁlisis DE CURVAS PARA EL DISEÑO DE MEZClAS DE CONCRETO CON MATERIAL TRITURADO DEL RÍO MAGDALENA EN EL SECTOR DE Girardot, Cundinamarca
}

\section{CURVE ANALYSIS FOR CONCRETE MIX DESIGN WITH SHREDDED

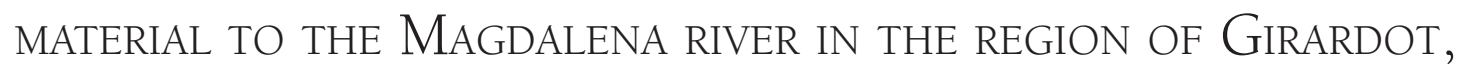 Cundinamarca}

\author{
Miguel Angel Avila Díaz* \\ Sandra Pinzón Galviss** \\ Luis Fernando Serna Hernández***
}

\section{RESUMEN}

Ell presente artículo exhibe un análisis de las curvas obtenidas para el diseño de mezclas de concreto, con material triturado del río Magdalena de la ciudad de Girardot-Cundinamarca. En este estudio se aplicó el método de diseño de mezclas A.C.I. 211, excluyendo la posibilidad de utilizar un agregado con una granulometría uniforme, debido a que la elaboración de estas mezclas en obras no cumple dicha característica. Para la obtención de las curvas se tomaron muestras de cilindros, arrojadas del diseño de 14

* Doctorando en Ciencias Ambientales de la Universidad del Valle-Cali. Estudios de maestría en Ciencias Químicas de la Universidad Nacional-Bogotá. Especialista en docencia universitaria de la Universidad Piloto de Colombia-Bogotá. Licenciado en Química de la Universidad Pedagógica Nacional-Bogotá. Docente-Investigador del Programa de Ingeniería Civil de la Universidad Piloto de Colombia-Seccional Alto Magdalena, Grupo de Investigación Desarrollo y Productividad en la Ciudad Región Girardot y el Alto Magdalena.

** Candidata a magíster en Gestión Urbana de la Universidad Piloto de Colombia-Bogotá. Ingeniera Civil de la Universidad Santo Tomás-Bogotá. Especialista en docencia universitaria de la Universidad Cooperativa-Ibagué. Docente-Investigadora del Programa de Ingeniería Civil de la Universidad Piloto de Colombia-Seccional Alto Magdalena, Grupo de Investigación Desarrollo y Productividad en la Ciudad Región Girardot y el Alto Magdalena.

*** Magíster en Administración de Empresas de la Universidad Viña del Mar-Chile. Especialista en gerencia de instituciones educativas y licenciado de Matemáticas de la Universidad del Tolima-Ibagué. Docente-investigador del Programa de Ingeniería Civil de la Universidad Piloto de Colombia-Seccional Alto Magdalena, Grupo de Investigación Desarrollo y Productividad en la Ciudad Región Girardot y el Alto Magdalena. 
mezclas de concreto, con agregados propios de la región; cada una de ellas con una relación A/C distinta, que varía en el rango de [0.35-0,60]; las cuales fueron probadas a compresión a los siete, 14 y 28 días. Este trabajo permitió refinar la curva de resistencia vs. relación agua/cemento con la que actualmente se cuenta, además de determinar las curvas de resistencia a los siete y 14 días proyectada a los 28 días, y la curva de resistencia vs. contenido de cemento; en procura de mejorar la calidad de las construcciones en la región al implementar metodologías de diseño adaptadas a las condiciones propias o materiales de la zona.

Palabras claves: concreto, resistencia, diseño de mezcla, relación agua/cemento

\begin{abstract}
This article shows an analysis of the curves obtained for the design of concrete mixtures with shredded material of the Magdalena River in the city of Girardot - Cundinamarca. The design method applied in this study was ACI 211 METHOD, excluding the possibility of using uniformly graded aggregate, because the preparation of these mixtures on site does not meet these specifications. In order to obtain the curves, cylinder samples were taken, reported by the design of 14 concrete mixes, which were made using aggregates typical to the region, each one of them with a different $\mathrm{A} / \mathrm{C}$ relation, varying in the range of $[0.35-0,60]$; which were tested by compression at 7,14 and 28 days. This work enabled to refine the curve of Resistance vs. Water / Cement ratio which is currently available, in addition to determining resistance curves at 7 and 14 days, Projected at 28 days, and the curve of Resistance vs. Cement Content; in an attempt to improve the quality of buildings in the region, to implement design methodologies tailored to the area materials and conditions.
\end{abstract}

Keywords: Concrete, Resistance, Mix design, Water /Cement ratio

\title{
INTRODUCCIÓN
}

En Colombia, la industria del concreto se ha difundido a tal punto que se ha convertido en uno de los principales materiales empleados en el campo de la construcción gracias a su gran versatilidad, dada por la facilidad que ofrece para ser vaciado en formaletas con casi cualquier forma arquitectónica durante su fase plástica.

El concreto es más económico de producir, comparado con algunos materiales frecuentemente utilizados en la construcción, ya que en el país hay abundante materia prima para su producción dada por la gran cantidad de ríos y canteras que existen en el territorio nacional. Además, se dispone de abundante mano de obra calificada, gracias a la tradición en su utilización, aumentando los beneficios económicos que se reciben al construir con éste material. 
Todo esto ha llevado a fomentar un mayor interés en el conocimiento del concreto por parte de los profesionales involucrados en ésta industria. En Colombia, se han realizado múltiples investigaciones por parte de distintas entidades interesadas en el conocimiento más profundo del comportamiento del concreto en los variados usos que se le dan a éste.

Existen en el país varias curvas y tablas en las cuales puede encontrarse la relación agua/cemento requerida para lograr una resistencia dada en un diseño de concreto. Sin embargo, para llegar a éstas se utilizaron materiales que pueden poseer propiedades físicas y mecánicas distintas a las de los materiales utilizados en nuestra región, como en nuestro caso de materiales extraídos del río Magdalena, propias del municipio de Girardot del departamento de Cundinamarca.

\section{METODOLOGÍA}

Para el desarrollo y culminación del estudio se utilizó el método A.C.I. 211, implementado para el diseño de mezclas en concreto, el cual permite obtener los datos específicos para modelar las curvas de resistencia a través de unos pasos definidos para la realización del diseño. En este sentido, se tuvo en cuenta las recomendaciones de dicho método, descartando la posibilidad de tener un agregado con una granulometría uniforme, debido a que el material empleado en la elaboración de este tipo de mezclas en obras no cumple con esta característica propia.

En consecuencia a esta metodología, se realizaron los siguientes ensayos a los materiales (arena y grava), necesarios para conocer los parámetros requeridos en el diseño de las mezclas:

1. *Granulometría: NTC 77

2. Peso unitario suelto: NTC 92

3. Peso unitario compactado: NTC 92

4. Contenido de materia orgánica: NTC 127

5. Pesos (peso específico saturado y superficialmente seco) grava: NTC176; arena: NTC 237

6. Absorción (agregado fino: NTC 237; agregado grueso: NTC 176)

7. Humedad natural: NTC 1776

*El ensayo de granulometría NTC 77 se descartó, porque esta característica no se cumple en obras.

Los pasos que se aplicaron según la metodología seleccionada para el proyecto, fueron:

i. Selección del asentamiento: Se optó por un asentamiento de $7,5 \mathrm{~cm}$, el cual es recomendado para estructuras comunes como vigas, fundiciones, columnas, muros y losas reforzadas y con compactación.

ii. Resistencia de diseño: Este parámetro no es fijo, sino que es un valor que se hallará como resultado de los ensayos a- compresión de los cilindros, sin esperar un valor determinado. 
iii. Relación A/C: Es el parámetro para dar inicio al diseño de mezclas y se asumirá con el fin de tener puntos bien distribuidos en el rango en el cual representa la curva buscada $[0,3-0, \boldsymbol{\theta}]$.

iv. Contenido de agua: Se toma como referencia la tabla del libro (Guzmán, 1987). Requerimiento aproximado de agua de mezclado para diferentes asentamientos y tamaños máximos de agregados, con partículas de forma redondeada y textura lisa en concreto, sin aire incluido.

v. Contenido de cemento: Teniendo en cuenta la relación A/C y el contenido de agua, se obtiene el contenido de cemento realizando la siguiente operación:

$C=\frac{A}{(A / C)}$

$C$ : Contenido de cemento, en $s / \mathrm{m}^{3}$

$A$ : Requerimiento de agua de mezclado en $/ \mathrm{m}^{3}$ (valor constante para todas las mezclas)

$A / C$ : Relación agua/cemento, por peso

vi. Volumen del agregado: Los diseños se realizan para $1 \mathrm{~m}^{3}$ de concreto, por lo tanto se deben conocer los volúmenes de agua, cemento y aire contenidos en la mezcla para obtener el volumen de agregados, como el faltante para completar el metro cúbico. Como ejemplo, se tiene:

V Cemento:

$$
492, \mathbf{B} s / \mathrm{m}^{3}\left(\mathrm{~m}^{3} / \mathrm{m}^{3}\right)
$$

V Agua: $188^{5} / \mathrm{m}^{3}$ se divide entre 1000 este valor para ser $\left(\mathrm{m}^{3} / \mathrm{m}^{3}\right)$ y peso específico $1000^{5} / \mathrm{m}^{3}\left(\mathrm{~m}^{3} / \mathrm{m}^{3}\right)$

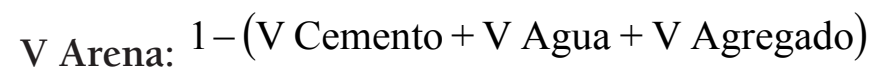

vii. Estimación del contenido de agregado grueso: El volumen absoluto del agregado grueso por volumen unitario de concreto se obtiene multiplicando el volumen seco y compactado por la relación entre la masa unitaria compacta y la densidad aparente seca del agregado:

$$
b=\left(b / b_{0}\right) * b_{0}
$$

$b$ :Volumen absoluto o sólido del agregado grueso, por unidad de volumen de concreto $b_{0}$ : Volumen absoluto o sólido del agregado grueso, por unidad de volumen compactado de agregado grueso

$b / b_{0}:$ (Guzman, 1987); y, 


$$
\begin{aligned}
& b_{0}=\frac{M A C}{D A S} \\
& M A C: \text { Masa unitaria compacta } \\
& D A S: \text { Densidad aparente seca }
\end{aligned}
$$

\section{OBTENCIÓN Y ANÁLISIS DE CURVAS}

A partir de la metodología implementada, se obtuvieron los datos necesarios para modelar algunas gráficas y curvas que permiten hacer una lectura descriptiva de la relación que existe entre la resistencia a los 28 días vs. la relación agua/cemento, la resistencia promedio a los siete días vs. la resistencia promedio a los 28 días, la resistencia promedio a los 14 días vs. la resistencia promedio a los 28 días y la cantidad de cemento vs. la resistencia promedio a los 28 días. En este sentido, en cada una de las gráficas se realiza un análisis de tendencia, con respecto a las variables independientes de los grafos.

Las gráficas son:

Figura 1. Curva de resistencia promedio a los 28 días vs. relación A/C

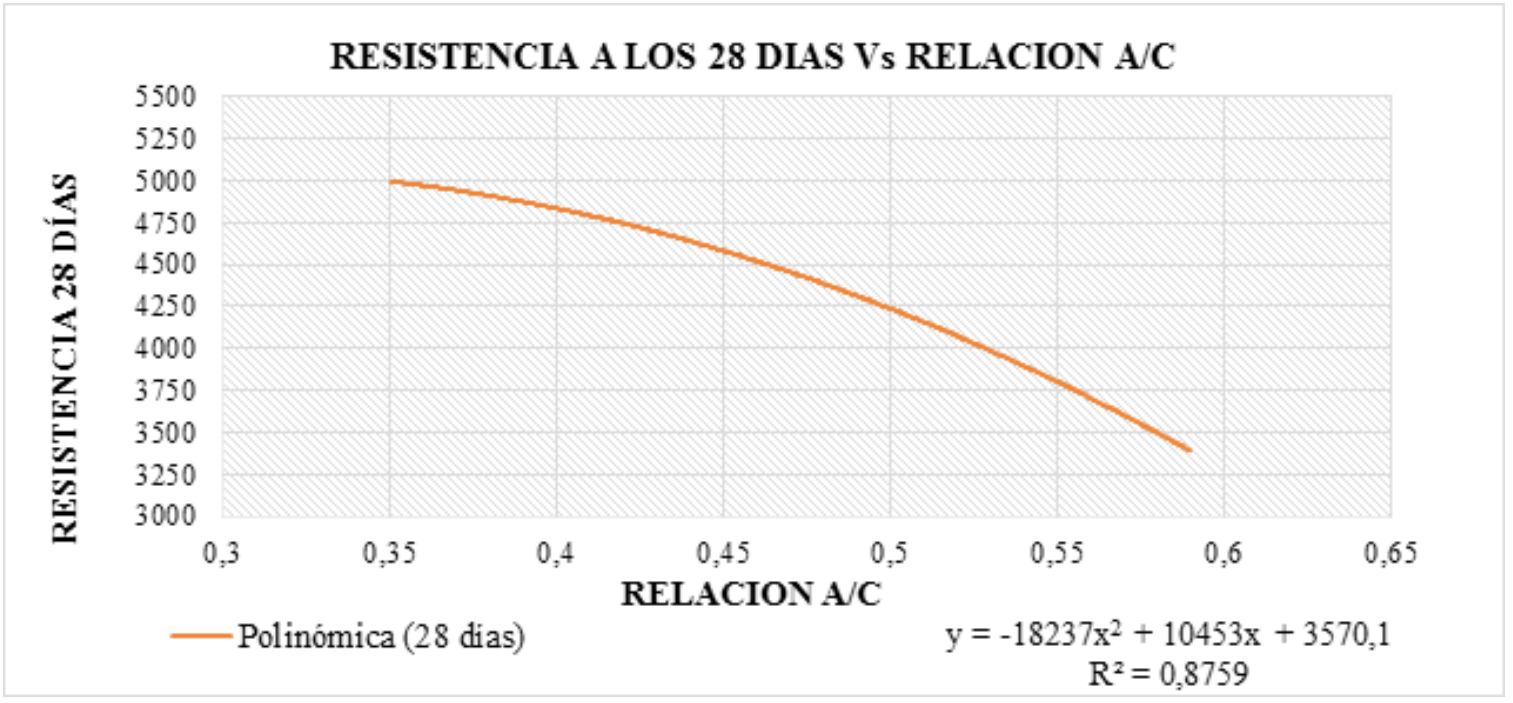

Fuente: Elaboración propia

Teniendo en cuenta la figura 1 , se evidencia que la relación agua/cemento se establece en una longitud de $0, \mathbb{Z} A / C$, permitiendo alcanzar una resistencia máxima de $5000 \mathrm{~b} / \mathrm{B}{ }^{2}$ en un rango de $[3400-5000]_{\mathrm{b}}^{\mathrm{b}} / \mathrm{B}^{2}$; también se puede diagnosticar que la resistencia proyectada a los 28 días disminuye a medida que la relación agua/cemento aumenta, generado a partir de una curva con puntos bien distribuidos en el rango de relación A/C $[0, \mathbf{3}-0, \boldsymbol{0}]$. 
Figura 2: Resistencia promedio a los 28 días vs. resistencia promedio a los siete dias

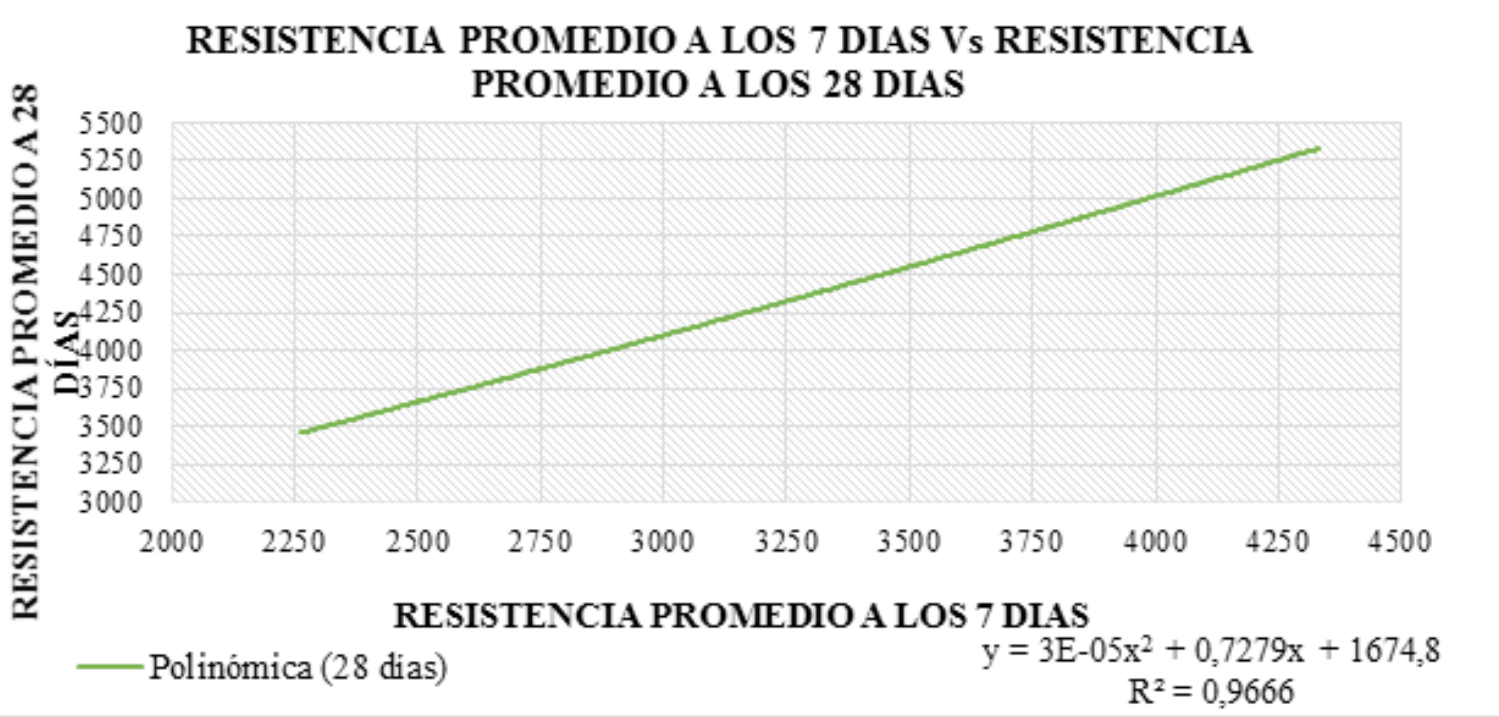

Fuente: Elaboración propia

La figura 2 evidencia la resistencia promedio a siete días, en un longitud de independencia de $2100 \mathrm{~b} / \mathrm{g}^{2}$ proyectada a la edad de 28 días, en un rango de $[3490-5400]_{\mathrm{b}} / \mathrm{g}^{2}$, logrando hacer un comparativo con respecto a su crecimiento. Es de resaltar la tendencia de crecimiento de esta función proporcional, que su incremento se expande de forma lineal a pesar que su ecuación polinómica está determinada por $y=3 e^{-\sigma} x^{2}+0,7279 x+1674,8$ , logrando mantener en equilibro las resistencias promedios a edades de siete a 28 días.

Figura 3. Resistencia promedio a los 28 días vs. resistencia promedio a los 14 días

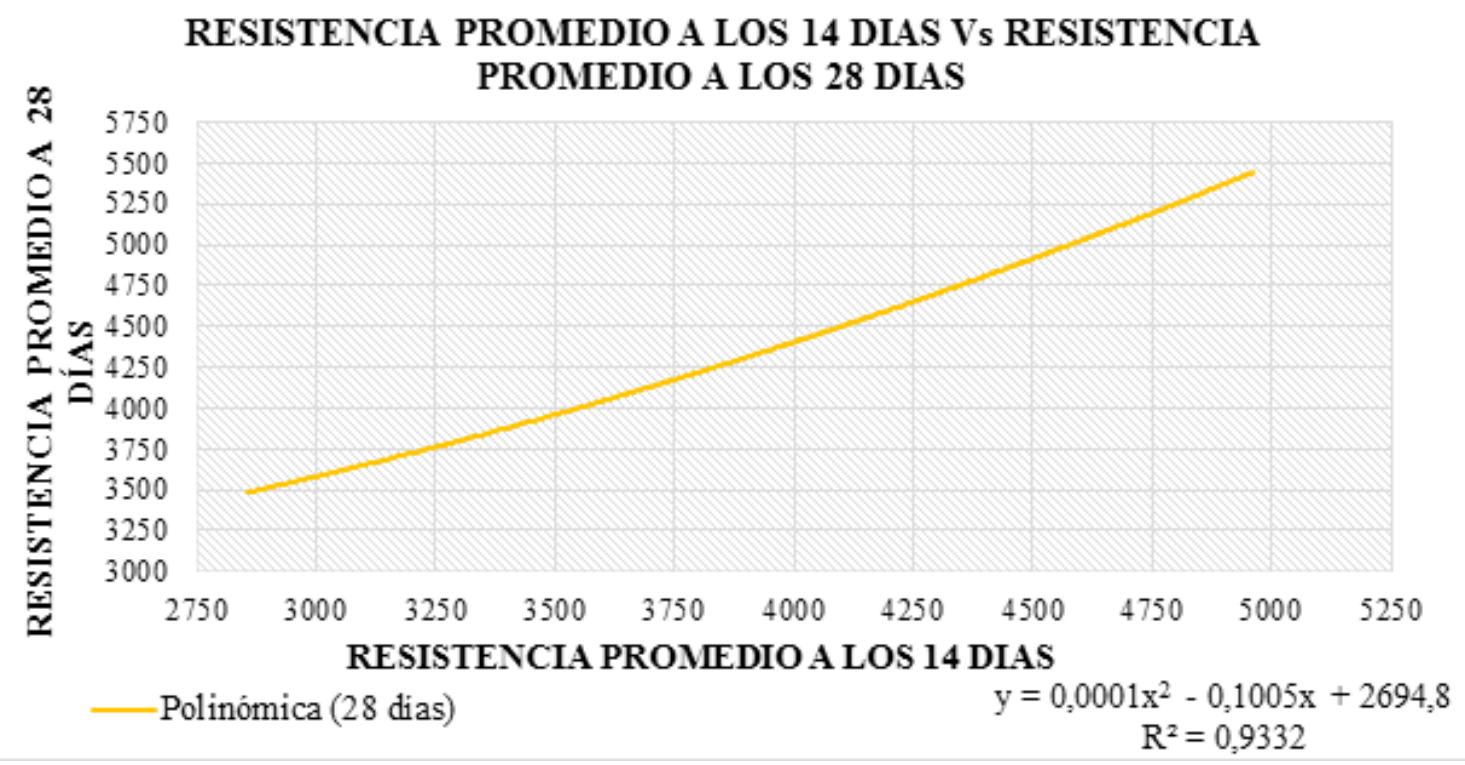

Fuente: Elaboración propia

En este sentido la figura 3, evidencia la resistencia a comprensión promedio a los 14 días, con una longitud de resistencia de $2100 \mathrm{~b} / \mathrm{B}{ }^{2}$ proyectada a la edad de 28 días en un 
rango de $[3490-5400]_{\mathrm{b}}^{\mathrm{b}} \mathrm{B}^{2}$, es de destacar la tendencia de crecimiento de esta función, debido a que su incremento se extiende de forma continua y creciente, manteniendo su directriz con respecto a la ecuación $y=0,0001 x^{2}-0,1005 x+2694,8$ y asimilando el mismo trayecto que la figura 2.

Figura 4. Resistencia promedio a los 28 dias vs. contenido de cemento

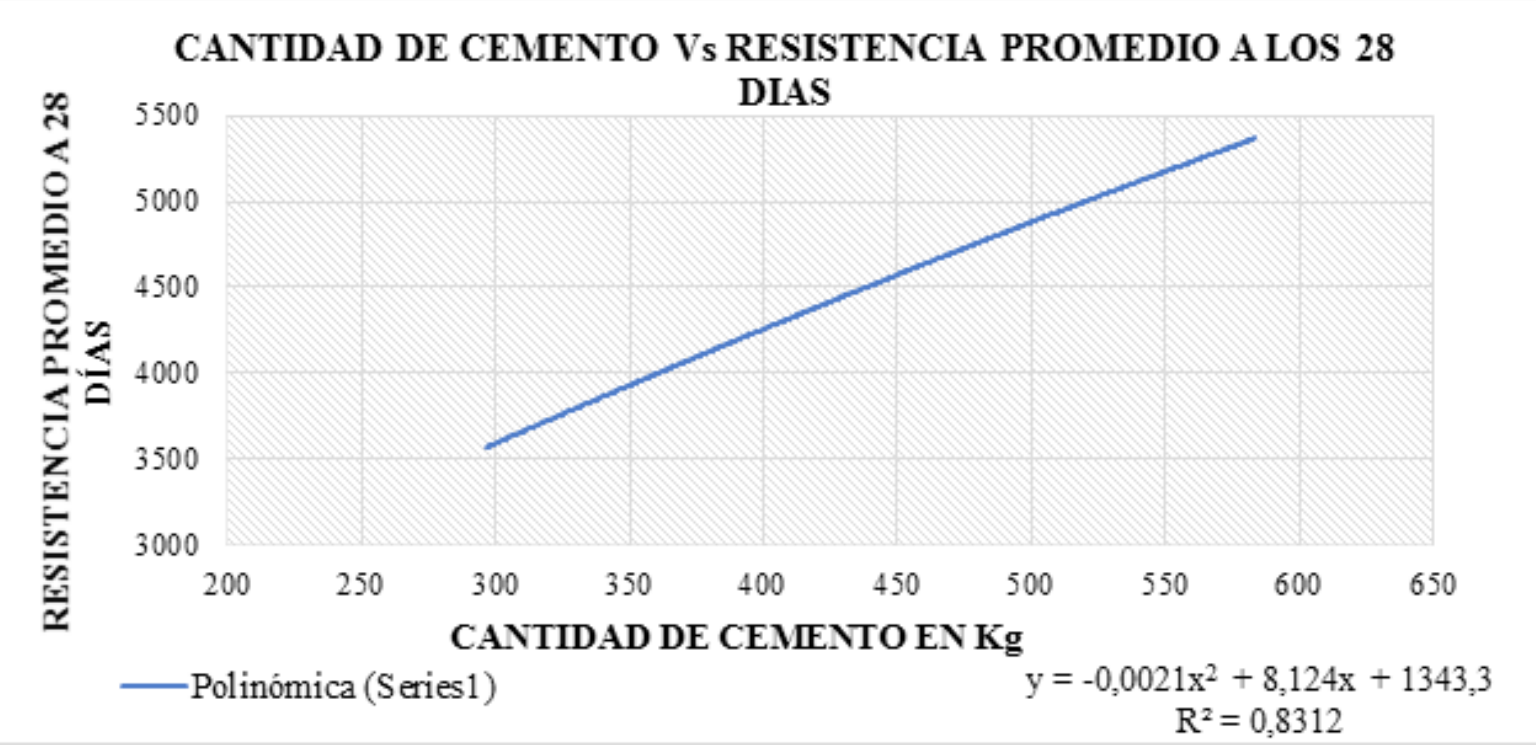

Fuente: Elaboración propia

Con relación a la figura 4, se evidencia la proporcionalidad de la curva con relación a la cantidad de cemento y la resistencia promedio a los 28 días, esto permite identificar una de las variables para mantener y aumentar la resistencia de concretos en tiempos proyectados, como se muestra en la figura. También se diagnostica los rangos de la resistencia con respecto a la cantidad de cemento suministrado en las pruebas y ensayos de cilindros de las mezclas de concreto, obteniendo un comparativo entre el cemento suministrado en una escala de [300 - 600] g y la resistencia futura alcanzada en un rango de $[3500-5400]^{b} / \mathrm{B}^{2}$.

\section{CONCLUSIONES}

Este trabajo establece que el concreto como material de construcción es demasiado versátil y práctico, además de económico, entre otras cualidades. Sin embargo, para que estas mismas características estén presentes se debe tener en cuenta la calidad de los agregados con lo que se piensa trabajar en obras de construcción, especialmente en el momento de diseñar la mezcla a utilizar; es por tal razón que será de gran ayuda lo estudiado y analizado en este proyecto, ya que establece las cualidades de los materiales del río Magdalena, muy comunes en la región del alto Magdalena donde se está desarrollando este proyecto. Se espera que a partir de este proceso de investigación, se generen e instauren bases sólidas a la comunidad del sector de la construcción a través de guías básicas para 
que se planifiquen los diferentes diseños de mezcla en concreto, ajustados a la economía y a las necesidades.

Según las diferentes graficas obtenidas en esta investigación, se puede observar la variación que se tiene en la relación agua/cemento, en las proyecciones a los siete, 14 y 28 días, lo cual es de gran importancia al momento de obtener las resistencias solicitadas por las especificaciones y normas establecidas con las proporciones justas, lo cual puede ahorrar tiempo y costos innecesarios.

Por lo anterior, es preciso dar la importancia real a estos diseños, ya que es evidente que al momento de realizar las mezclas en concreto en sitio de obra no se tiene ninguna clase de control, por lo cual las relaciones varían y se obtienen concretos con características no apropiadas, baja de calidad o a costos elevados.

Todo lo anterior resalta la importancia de investigaciones como esta para la región, en procura de mejorar la calidad de sus construcciones al utilizar metodologías de diseño cada vez menos variables, puesto que aunque se apliquen métodos foráneos, sus parámetros pueden ser modificados para adaptarlos a las condiciones propias de la zona.

En las demás curvas presentadas en las figuras 2 y 3, puede encontrarse un elemento de gran importancia, especialmente para el control de calidad que se le realiza al concreto a lo largo de su etapa de fraguado, que normalmente se sigue hasta los 28 días. Con estas curvas elaboradas con suficiente confiabilidad estadística, podría conocerse la resistencia a los 28 días del concreto desde temprano y así permitir, en caso de cualquier falla, tomar los correctivos pertinentes con mayor anticipación.

\section{REFERENCIAS BIBLIOGRÁFICAS}

Arias Vásquez, L. F. (1999). Curva de resistencia vs. Relación agua/cemento para el diseños de mezclas regionales. Manizales: Universidad Nacional de Colombia.

Galvis Ramos, J. (1988). Diseño de mezclas en concreto para la ciudad de Manizales. Manizales: Universidad Nacional de Colombia.

Gutiérrez de López, L. (1996). El concreto y otros materiales para la construcción. Manizales: Universidad Nacional de Colombia.

León Cárdenas, H. D. y Morales Castro, J. A. (2000). Refinamiento de la curva de relación agua/cemento vs. resistencia para el diseño de mezclas regionales. Manizales: Universidad Nacional de Colombia.

Matallana Rodríguez, R. (S. F.). Concreto: Serie de conocimientos básicos. Bogotá: Instituto Colombiano de Productores de Cemento y Asocreto.

Mattheib, J. (1980). Hormigón armado aligerado pretensado. Barcelona: Reverte S. A. 
Sánchez de Guzmán, D. (2001). Tecnología del concreto y del mortero. 5. a ed. Bogotá: Bhandar editores Ltda.

Sandino Pardo, A. (1988). Tecnología del concreto. Primera ed. Bogotá: Asociación de ingenieros civiles. 